\title{
Is bigger better: The association between follicle size and livebirth rate following IVF?
}

\author{
Jaime M. Knopman*, James A. Grifo, Akiva P. Novetsky, Meghan B. Smith, Alan S. Berkeley
}

NYU Fertility Center, NYU Langone School of Medicine, New York, USA

Email: *jaimeknopman@gmail.com

Received 26 September 2012; revised 27 October 2012; accepted 6 November 2012

\begin{abstract}
Purpose: To analyze the correlation between lead follicle size at hCG trigger and IVF outcome. Methods: A review of all patients undergoing their first IVF cycle between 1/2005-12/2009 was performed. Four groups were evaluated $(<18 \mathrm{~mm}, 18-18.9 \mathrm{~mm}$, 19 - $19.9 \mathrm{~mm}$ and $\geq 20 \mathrm{~mm}$ ), based on the mean diameter of the two largest follicles on day of ovulation trigger (OT); cycle parameters and outcomes were analyzed. Results: 1577 cycles were reviewed. There were no significant differences noted for cycle parameters or outcomes for the 4 groups. However, although LBR was not significantly different, there was a decline noted as lead follicle size increased. Conclusions: Delaying the administration of OT to enhance follicular growth does not appear to improve IVF outcome. Larger lead follicles do not yield a higher percentage of mature oocytes, embryos available for transfer or LBR. A misguided zest to achieve a higher quantity of fertilizable oocytes may impair oocyte and embryo quality.
\end{abstract}

Keywords: Folliculogenesis; Oocyte Maturity; Timing of Ovulation Trigger; Follicular Size and IVF Outcome

\section{INTRODUCTION}

The success of IVF hinges on the ability to obtain an adequate number of mature oocytes that subsequently develop into competent embryos. Identifying the follicle size that will yield such oocytes can be challenging. Therefore, designating an appropriate time for hCG administration, a decision which is often largely based on follicular size, remains controversial. While some studies in the literature suggest that oocytes from larger follicles yield superior oocytes with improved fertilization and implantation potential, other studies report that delaying ooctye retrieval neither increases the number of mature oocytes retrieved nor the pregnancy rate and may, in fact,

"Corresponding author. impair embryo quality [1-24].

Since the inception of IVF, there has been a progression in how ovarian stimulation is managed; in the early days larger follicles were required because this correlated with oocyte maturity in the natural cycle as well as in a clomiphene stimulated cycle [6,7,12-15]. However, the introduction of injectable gonadotropins to achieve multi-follicular development resulted in a paradigm shift in the goal of IVF; multiple large follicles yielding multiple fertilizable oocytes supplanted a desire to achieve one fertilizable oocyte [20]. This push for "more" significantly increased the number of oocytes retrieved as well as IVF success rates, but came with the cost of an increase in cycle cancellations due to premature LH surges $[6,15]$. In an effort to avoid cycle failures, ovulation trigger was administered earlier translating into smaller lead follicle sizes. Their results demonstrated that administering hCG in gonadotropin stimulated cycles when at least two follicles were $16 \mathrm{~mm}$ in diameter increased the probability of achieving a pregnancy [16]. The addition of GnRH agonists and antagonists significantly reduced the incidence of premature LH surges and cycle cancellations and allowed practitioners to delay hCG trigger to achieve larger multi-follicular growth, which ultimately translated to a higher number of oocytes retrieved and improved pregnancy rates. Therefore, many IVF programs elected to prolong follicular stimulation to achieve larger lead follicle sizes, similar to those seen when natural and clomiphene stimulated IVF cycles were routine, with the hope that this delay would yield a higher number of mature fertilizable oocytes capable of developing into competent embryos and better pregnancy rates. The assumption became that bigger lead follicles yielded better pregnancy rates $[18,19,21,22]$.

Traditionally, our program administered hCG at relatively small sizes; when the 2 lead follicles reached $17 \mathrm{~mm}$, ovulation trigger was given. Over time as additional ART procedures became available, specifically, the microdose lupron protocol, pre-implantation genetic diagnosis and oocyte cryopreservation, the doctrine that 
larger follicles were needed to attain a higher percentage of mature oocytes in these particular instances began to infiltrate our practice and gradually changed the size at which practitioners were administering hCG (even for first time IVF cycles without any of such procedures). Therefore, the objective of our study was to identify if prolonging follicular stimulation to achieve increased follicular growth increased the number of oocytes retrieved, the percent of mature oocytes, the CPR and LBR.

\section{MATERIALS AND METHODS}

All patients who underwent and completed their first IVF cycle ever between $1 / 2005$ to $12 / 2009$, were analyzed retrospectively. Patients who had undergone a previous IVF cycle either at our institution or elsewhere were excluded. These patients were selected to limit bias as there would be no information available regarding follicle size and oocyte maturity. In addition, all donor oocyte cycles, oocyte cryopreservation cycles and ovulation induction cycles converted to IVF were excluded from our analysis. Of note, our center does not permit any patient to start a IVF cycle during the month with an unsuppressed day 2 serum FSH $>13.5 \mathrm{IU} / \mathrm{L}$ or E2 $>80$ $\mathrm{pg} / \mathrm{mL}$.

Patients who met the inclusion criteria were categorized based on the mean diameter of the two largest follicles recorded on the day of ovulation trigger (OT); $<18 \mathrm{~mm}, 18-18.9 \mathrm{~mm}, 19-19.9 \mathrm{~mm}, \geq 20 \mathrm{~mm}$. All ultrasounds were performed by any one of five attending physicians or one of seven fellows with a similar technique of measuring the mean maximum diameter in two dimensions of each follicle.

Stimulation was performed using a variety of standard protocols (luteal GnRH agonist, GnRH antagonist protocol or microdose GnRH agonist). Stimulation protocol and starting gonadotropin dosage was determined by a combination of factors including patient age, ovarian reserve testing, and previous response to ovulation induction/intra-uterine insemination cycles (if applicable); dosage was subsequently adjusted according to individual response. Ultrasound and estradiol monitoring were performed periodically (frequency based on patient response to medications) throughout the IVF cycle. In all protocols 10,000 units of units of IM hCG were administered when at least two lead follicles measured 17 $\mathrm{mm}$ in diameter. The two largest follicles recorded on the day of OT were recorded; the mean diameter of these two follicles were calculated and used to categorize patients into one of the four study groups.

Oocyte retrieval by ultrasound guided transvaginal aspiration occurred 34 - 36 hours after hCG administration. Oocytes were collected from follicular fluid and immediately placed in $75-\mu \mathrm{L}$ droplets of human tubal fluid (HTF: Irving Scientific, Irvine, CA) supplemented with 6\% Plasmanate (plasma protein fraction 5\%; USP, Bayer Co., Elkhart, IN) overlaid with Sage mineral oil (Cooper Surgical Co., Trumbull, CT). Partner's sperm was collected on day of oocyte retrieval and washed. Oocytes were fertilized with routine insemination at $4-6$ hours after retrieval or ICSI was performed when indicated for severe male factor or a prior history of poor fertilization. Oocytes inseminated by ICSI were transferred to Quinn's advantage cleavage media (Q1) (Cooper Surgical) supplemented by $10 \%$ Plasmanate. Oocyte maturity was determined by the presence or absence of the polar body approximately 18 hours after oocyte retrieval. Fertilization was assessed 18 hours after insemination or ICSI by visualization of two pronuclei (2PN). Embryos that fertilized after standard insemination were then transferred to Quinn's advantage cleavage media supplemented by $10 \%$ Plasmanate. Embryos were incubated and monitored daily for blastomere number, symmetry, degree of fragmentation and overall quality. Good quality embryos on day 3 after oocyte retrieval were defined as $>5$ cells with a grade better than 2.5 . Embryos that were still dividing were transferred to Quinn's advantage blastocyst (Q2) media supplemented with $10 \%$ Plasmanate and cultured to day 5 . The decision to culture to day 5 was based on quantity and quality of embryos on day 3 . If an excess number of good quality embryos are available for transfer (generally $\geq 3 \mathrm{em}-$ bryos), then embryo culture would be continued. Embryos were graded on day 5 using the Gardner and Lane criteria. All embryo transfers were performed under direct ultrasound guidance. The number of 2 pn zygotes as well as blastocysts were recorded and analyzed. All patients received daily IM P (50 mg in sesame oil) for luteal support. If the pregnancy test on day 28 (14 days after retrieval) was positive, then the patient continued daily injections of P. Monitoring with B-hCG levels and trans-vaginal ultrasounds were performed to identify viable intra-uterine pregnancies.

The study groups were analyzed for differences in clinically relevant variables. In patients suppressed using leuprolide acetate and/or oral contraceptives treatment cycle day $2 / 3$ serum FSH were not available and therefore baseline ovarian reserve testing was used for analysis.

Categorical variables in the study groups were compared by chi-squared analyses. Kruskal-Wallis analysis of variance was used for comparison of not normally distributed continuous variables. A p-value of $<0.05$ was considered statistically significant. A sub-group analysis of all patients in group 4 (mean follicular diameter $\geq 20$ $\mathrm{mm}$ ) was performed based on the day that ovulation trigger was administered. In addition, results were further analyzed based on protocol. All statistical analyses were 
performed using Stata 9.0 (College Station, TX).

\section{RESULTS}

In total, 1577 first time IVF cycles were analyzed. Patients were assigned to four groups; $<18 \mathrm{~mm}, 18-18.9$ $\mathrm{mm}, 19-19.9 \mathrm{~mm}, \geq 20 \mathrm{~mm}$, based on the mean diameter of the two largest follicles measured and recorded on the day of OT. There were no differences in baseline demographics or cycle parameters between the four groups (Table 1).

No significant differences were noted in cycle outcomes between the four groups (Table 2). However, the number of oocytes retrieved approached significance $(p=0.06)$, with those in study group 1 (mean follicular diameter $<18 \mathrm{~mm}$ ) having the highest number of oocytes retrieved. Although the CPR and LBR were not significantly different between the four study groups $(\mathrm{p}=$ 0.57 and $p=0.62$, respectively), there was a non-significant decline in LBR as the lead follicle size increased.

A sub-analysis of the patients in study group 4 (mean diameter of the 2 lead follicles $>20 \mathrm{~mm}$ ) was performed. The patients were divided into 3 groups; those who received OT on or before day 10 , those who received OT on days 11 or 12 and those who received OT on day 13 or greater (Table 3). This sub-group analysis was performed to compare those patients who reached large follicles early, those who reached large follicle size on the average day of OT for the entire group and for those patients who were pushed past the average day of OT to achieve marked follicular growth. A significant difference between the day 2 FSH values and the total gonadotropin dosage administered was noted $(\mathrm{p}<0.0001$ for both). Earlier trigger was associated with higher FSH value and lower doses of administered gonadotropins. Despite the prolonged follicular stimulation, group 3 did not have a higher number of oocytes retrieved, a higher percentage of mature oocytes, a higher embryo progression to blastocyst, or a higher CPR or LBR.

The data was further analyzed by method of pituitary suppression (Tables 4 and 5). In those patients receiving a GnRH antagonist protocol $(\mathrm{n}=933)$, there was a significant difference noted among the 4 groups with regard to patient age and the cycle day of OT without any significant differences in cycle outcomes Table 5). In those patients receiving a long GsnRH agonist protocol $(\mathrm{n}=571)$, women who received OT at the smallest lead follicle size $(<18 \mathrm{~mm})$ had significantly more oocytes retrieved $(\mathrm{p}<0.008)$ without any sig-

Table 1. Patient demographics/cycle parameters ${ }^{*}$.

\begin{tabular}{|c|c|c|c|c|c|}
\hline $\mathrm{N}=1577$ & $\begin{array}{c}\text { Group 1 } \\
<18 \mathrm{~mm}(\mathrm{n}=360)\end{array}$ & $\begin{array}{c}\text { Group 2 } \\
18-18.9 \mathrm{~mm}(\mathrm{n}=559)\end{array}$ & $\begin{array}{c}\text { Group 3 } \\
19-19.9 \mathrm{~mm}(\mathrm{n}=373)\end{array}$ & $\begin{array}{c}\text { Group 4 } \\
\geq 20 \mathrm{~mm}(\mathrm{n}=285)\end{array}$ & $\mathrm{P}$ value \\
\hline Patient Age (y) & $36(33-40)$ & $36(33-39)$ & $33(33-39)$ & $36(32-39)$ & 0.37 \\
\hline Day 2 FSH (IU/L) & $6.5(4.8-8)$ & $6.7(5-8.2)$ & $6.7(5.2-8.6)$ & $6.5(4.9-8.6)$ & 0.25 \\
\hline Total Gonadotropin Dose (IU) & $2625(1850-3600)$ & $2700(1950-3600)$ & $2750(2025-3600)$ & $2712(2025-3750)$ & 0.58 \\
\hline Serum E2 at OT $(\mathrm{pg} / \mathrm{mL})$ & $1758(1094-2782)$ & $1742(1126-2561)$ & $1748(1179-2562)$ & $1792(1177-2523)$ & 0.94 \\
\hline
\end{tabular}

*All data are reported as median values (interquartile range); OT = ovulation trigger.

Table 2. IVF cycle outcomes ${ }^{*}$.

\begin{tabular}{|c|c|c|c|c|c|}
\hline $\mathrm{N}=1577$ & $\begin{array}{c}\text { Group 1 } \\
<18 \mathrm{~mm}(\mathrm{n}=360)\end{array}$ & $\begin{array}{c}\text { Group 2 } \\
18-18.9 \mathrm{~mm}(\mathrm{n}=559)\end{array}$ & $\begin{array}{c}\text { Group 3 } \\
19-19.9 \mathrm{~mm}(\mathrm{n}=373)\end{array}$ & $\begin{array}{c}\text { Group 4 } \\
\geq 20 \mathrm{~mm}(\mathrm{n}=285)\end{array}$ & $\mathrm{P}$ value \\
\hline \#Eggs Retrieved & $14(9-21)$ & $13(9-19)$ & $13(8-19)$ & $12(8-18)$ & 0.06 \\
\hline \#MII & $11(7-17)$ & $11(7-16)$ & $11(7-16)$ & $10(7-14)$ & 0.12 \\
\hline$\% \mathrm{MII}$ & $86(75-97)$ & $86(75-100)$ & $88(76-100)$ & $88(74-100)$ & 0.62 \\
\hline \#2pn-zyogtes & $8(5-12)$ & $8(4-12)$ & $8(5-13)$ & $7(4-11)$ & 0.35 \\
\hline \#Embryos Transferred & $2(2-2)$ & $2(2-2)$ & $2(2-2)$ & $2(2-2)$ & 0.91 \\
\hline \#Blastocysts & $5(1-8)$ & $5(1-9)$ & $5(2-8)$ & $4(1-7)$ & 0.46 \\
\hline Clinical Pregnancy Rate & $53.3 \%$ & $49.6 \%$ & $50.9 \%$ & $48.1 \%$ & 0.57 \\
\hline Livebirth Rate & $45.6 \%$ & $43.7 \%$ & $43.2 \%$ & $40.4 \%$ & 0.62 \\
\hline
\end{tabular}

*All data are reported as median values (interquartile range). 
Table 3. Group 4 sub-analysis based on the day of $\mathrm{OT}^{* \dagger}$.

\begin{tabular}{|c|c|c|c|c|}
\hline $\mathrm{N}=285$ & Day $\leq 10(\mathrm{n}=96)$ & Day $11-12(\mathrm{n}=107)$ & Day $\geq 13(n=82)$ & $P$ value \\
\hline Age (y) & $37(33-39)$ & $36(32-39)$ & $36(31-40)$ & 0.31 \\
\hline Day 2 FSH (IU/L) & $7.35(6-9.2)$ & $6.7(5.1-9.6)$ & $5(2-6.8)$ & $<0.0001$ \\
\hline Total Gonadotropin Dose (IU) & $2350(1612.5-3075)$ & $2700(2025-3750)$ & $3825(2663-4950)$ & $<0.0001$ \\
\hline Serum E2 at OT (pg/mL) & $1604.5(1144-2423)$ & $1886(1215-2634)$ & $1799.5(1247-2802)$ & 0.14 \\
\hline \#Eggs Retrieved & $11(8-15)$ & $14(9-19)$ & $12(8-19)$ & 0.06 \\
\hline$\%$ MII & $86(72-100)$ & $86(74-100)$ & $90(76-100)$ & 0.34 \\
\hline \#2pn-zygotes & $7(4-10.5)$ & $8(4-12)$ & $7(5-10)$ & 0.51 \\
\hline \#Blastocysts & $4(1.5-7)$ & $4(1-8)$ & $4(1-7)$ & 0.89 \\
\hline Clinical Pregnancy Rate & $51.0 \%$ & $47.7 \%$ & $45.1 \%$ & 0.73 \\
\hline Livebirth Rate & $43.8 \%$ & $37.4 \%$ & $40.2 \%$ & 0.65 \\
\hline
\end{tabular}

*All data are reported as median values (interquartile range); ${ }^{\dagger}$ Group 4 included all patients where the mean diameter of the 2 lead follicles was $>20 \mathrm{~mm}$.

Table 4. Patients receiving GnRH antagonist protocol*.

\begin{tabular}{cccccc}
\hline N=933 & $<18 \mathrm{~mm}(\mathrm{n}=186)$ & $18-18.9 \mathrm{~mm}(\mathrm{n}=314)$ & $19-19.9 \mathrm{~mm}(\mathrm{n}=240)$ & $\geq 20 \mathrm{~mm}(\mathrm{n}=193)$ & P value \\
\hline Age (y) & $38(35-41)$ & $38(34-41)$ & $37(34-41)$ & $37(33-40)$ & 0.02 \\
Day 2 FSH (IU/L) & $7(5.2-9)$ & $7(5.1-8.9)$ & $7.4(5.5-9.1)$ & $6.9(5.3-9.2)$ & 0.82 \\
Total Gonadotropin Dose (IU) & $2928(2075-390)$ & $2775(2025-3600)$ & $2948(2025-3600)$ & $2712(2025-3600)$ & 0.75 \\
Cycle day of OT & $10(9-12)$ & $10(10-12)$ & $11(10-12)$ & $11(10-12)$ & 0.02 \\
Serum E2 at OT (pg/mL) & $1391(998-2159)$ & $1399(1051-2164)$ & $1567.5(1099.5-2293)$ & $1692(1166-2358)$ & 0.14 \\
\#Eggs Retrieved & $12(8-20)$ & $12(8-19)$ & $12(8-17)$ & $12(8-17)$ & 0.79 \\
\%MII & $86(75-97)$ & $86(75-100)$ & $88.5(76-100)$ & $88(75-100)$ & 0.55 \\
\#2pn-zygotes & $7(4-11)$ & $7(4-12)$ & $7(4-11)$ & $7(4-10)$ & 0.90 \\
\#Blastocysts & $4(0-7)$ & $4(1-8)$ & $4(1-8)$ & $4(1-7)$ & 0.63 \\
Clinical Pregnancy Rate & $50.30 \%$ & $44.90 \%$ & $39.30 \%$ & $45.10 \%$ & 0.67 \\
Livebirth Rate & $43.20 \%$ & $37.90 \%$ & & $37.80 \%$ & 0.99 \\
\hline
\end{tabular}

*All data are reported as median values (interquartile range).

nificant differences noted in clinical outcomes.

\section{DISCUSSION}

Ovulation trigger should not be postponed to achieve increased follicular size or improve outcomes in patients undergoing their first IVF cycle. This practice increases patient cost and time without increasing the number of oocytes retrieved, the percentage of mature oocytes, the number of embryos available for transfer or the live birth rate. Furthermore, in our study, the highest number of oocytes retrieved was from those patients with the smallest lead follicles, defying the concept that bigger lead follicles yield a higher quantity of oocytes. In addition, although not significantly different, as the mean diameter of the lead follicle increased there was a slow but steady decline in the LBR as the lead follicle size increased, suggesting that larger follicles may have a negative impact on IVF outcome.

Why larger lead follicles not only failed to yield a higher percentage of mature oocytes but also resulted in a lower, albeit not significantly so, live birth rate is not clear. One plausible explanation can be offered by recent data; oocyte maturity is an intricate process that is not simply defined by the presence of the 2nd polar body. Rather, it requires "nuclear maturity" (defined by the presence of the 2nd polar body) as well as cytoplasmic maturity (defined by the appearance of the cumulus-corona complex and the cytoplasm) $[1,4,9,10]$. The completion of both events is necessary to the formation 
Table 5. Patients receiving long GnRH agonist protocol*.

\begin{tabular}{cccccc}
\hline N $=571$ & $<18 \mathrm{~mm}(\mathrm{n}=155)$ & $18-18.9 \mathrm{~mm}(\mathrm{n}=221)$ & $19-19.9 \mathrm{~mm}(\mathrm{n}=117)$ & $\geq 20 \mathrm{~mm}(\mathrm{n}=78)$ & P value \\
\hline Age (y) & $34(31-37)$ & $34(32-38)$ & $34(31-37)$ & $33(30-37)$ & 0.20 \\
Day 2 FSH (IU/L) & $6.15(4.9-7.2)$ & $6.3(4.9-7.5)$ & $6.2(4.8-7.2)$ & $6(4.8-6.95)$ & 0.73 \\
Total Gonadotropin Dose (IU) & $2050(1650-3000)$ & $2400(1850-3075)$ & $2400(1950-3150)$ & $2400(1875-3600)$ & 0.11 \\
Cycle day of OT & $12(11-13)$ & $12(11-13)$ & $12(11-13)$ & $12(11-13)$ & 0.12 \\
Serum E2 at OT (pg/mL) & $2554(1526-3375)$ & $2284(1517.5-2991)$ & $2246(1614-3297)$ & $2192.5(1520-3375)$ & 0.44 \\
\#Eggs Retrieved & $18(12-25)$ & $15(10-20)$ & $16(11-23)$ & $14(9-20)$ & 0.008 \\
\% MII & $86(75-94)$ & $85(75-94)$ & $86(75-95)$ & $88.5(74-96)$ & 0.91 \\
\#2pn-zygotes & $10(6-15)$ & $9(6-13)$ & $9(5-14)$ & $7(5-13)$ & 0.16 \\
\#Blastocysts & $6(4-9)$ & $6(3-10)$ & $6(4-10)$ & $5(3-9)$ & 0.49 \\
Clinical Pregnancy Rate & $60.70 \%$ & $56.80 \%$ & $63.30 \%$ & $53.00 \%$ & $51.30 \%$ \\
Livebirth Rate & $51.60 \%$ & $52.70 \%$ & & 0.67 \\
\hline
\end{tabular}

*All data are reported as median values (interquartile range).

of an oocyte that is capable of developing into a competent embryo. Prolonged follicular stimulation may tamper with these processes damaging a cohort of oocytes and decreasing the likelihood of IVF success.

There are several limitations to our study. First, while all follicular measurements were done in one fertility center, there were several different physicians performing the ultrasounds. Although one technique was employed by all practitioners to measure follicular diameter, inherent variability exists between physicians. Such subjectivity could have reduced the accuracy of the measurements recorded. Second, all first-time IVF patients (regardless of IVF protocol) were included in our analysis. Although we attempted to control for this confounder by analyzing the two most commonly prescribed protocols separately, because of small numbers we did not assess all protocols individually (ex; microdose lupron). While such patients made up a very small percent of the patients studied, their inclusion in our study group may have affected our results. Third, infertility diagnoses were not captured or analyzed in our review and therefore the impact of infertility diagnoses on follicular size and IVF outcome was not assessed. Although a correlation between such parameters and ovarian stimulation has not been clearly identified, specific diagnoses could have an impact on IVF outcome and the validity of our results. Of note, because baseline ovarian reserve can have a significant impact on ovarian stimulation we compared day 2 FSH values; no significant differences were noted between the four study groups. Fourth, it is not our practice to routinely collect progesterone levels on the day of hCG administration. It is possible that such data would demonstrate abnormal progesterone values in those patients with larger follicles suggesting premature luteinization occurred despite the use of pituitary suppression. Lastly, our review was retrospective, in nature thereby limiting our ability to control for confounding factors.

In summary, follicular development and its relationship to oocyte competence is an intricate process. While lead follicle size may predict oocyte maturity and therefore advanced follicular growth should theoretically yield an increased number of mature oocytes, delaying OT may not improve oocyte quality and may in fact have a negative impact on the oocyte and its resultant embryos ability to progress and ultimately yield a baby. Thus, the zest to increase the quantity of fertilizable oocytes by creating bigger follicles appears to be a flawed theory and this practice may result in diminished ooctye and embryo quality.

\section{REFERENCES}

[1] Balaban, B. and Urman, B. (2006) Effect of oocyte morphology on embryo development and implantation. Reproductive BioMedicine Online, 12, 608-615. doi:10.1016/S1472-6483(10)61187-X

[2] Ben-Rafael, Z., Kopf, G.S., Blasco, L., Flickinger, G.L., Tureck, R.W., Strauss, J.F. and Mastroianni, L. (1986) Follicular maturation parameters associated with the failure of oocyte retrieval, fertilization and cleavage in vitro. Fertility and Sterility, 45, 51-57.

[3] Bergh, C., Broden, H., Lundin, K. and Hamberger, L. (1998) Comparison of fertilization, cleavaage and pregnancy rates of oocytes from large and small follicles. Human Reproduction, 13, 1912-1915. doi:10.1093/humrep/13.7.1912 
[4] Ciotti, P.M., Notarangelo, L., Morselli-Labate, A.M., Felletti, V., Porcu, E. and Venturoli, S. (2004) First polar body morphology before ICSI is not related to embryo quality or pregnancy rate. Human Reproduction, 19, 2334-2339. doi:10.1093/humrep/deh433

[5] Clark, L., Stanger, J. and Brinsmead, M. (1991) Prolonged follicular stimulation decreases pregnancy rates after in vitro fertilization. Fertility and Sterility, 55, 11921194.

[6] DeCherney, A.H., Tarlatzis, B.C. and Laufer, N. (1985) Follicular development: Lessons learned from human IVF. American Journal of Obstetrics \& Gynecology, 153, 911923.

[7] Droesch, K., Muasher, S.J., Kreiner, D., Jones, G.S., Acosta, A.A. and Rosenwaks, R. (1988) Timing of oocyte retrieval in cycles with a spontaneous luteinizing hormone surge in a large in vitro fertilization program. Fertility and Sterility, 50, 451-456.

[8] Dubey, A.K., Wang, H.A., Duffy, P. and Penzias, A. (1995) The correlation between follicular measurements, oocyte morphology, and fertilization rates in an in vitro fertilization program. Fertility and Sterility, 64, 787-790.

[9] Ebner, T., Moser, M., Sommergruber, M., Puchner, M., Wiesinger, R. and Tews, G. (2003) Developmental competence of oocytes showing increased cytoplasmic viscosity. Human Reproduction, 18, 1294-1298. doi:10.1093/humrep/deg232

[10] Ebner, T., Moser, M., Sommergruber, M., Gaiswinkler, U., Shebl, O., Jesacher, K. and Tews, G. (2005) Occurrence and developmental consequences of vacuoles throughout preimplantation development. Fertility and Sterility, 83, 1635-1640. doi:10.1016/j.fertnstert.2005.02.009

[11] Ectors, F.J., Vanderzwalmen, P., Van Hoeck, J., Nijs, M., Verhaegen, G., Delvigne, A., Schoysman, R. and Leroy, F. (1997) Relationship of human follicular diameter with oocyte fertilization and development after in-vitro fertilization of intracytoplasmic sperm injection. Human Reproduction, 12, 2002-2005.

[12] Edwards, R.G. (1973) Physiological aspects of human ovulation, fertilization and cleavage. Journal of Reproduction and Fertility. Supplement, 18, 87-101.

[13] Edwards, R.G. and Steptoe, P.C. (1975) Induction of follicular growth ovulation and luteinization in the human ovary. Journal of Reproduction and Fertility. Supplement, 22, 121-160.

[14] Garcia, J., Acosta, A., Andrews, M.C., Jones, G.S., Jones, H.W., Mantzavinos, T., Mayer, J., McDowell, J., Sandow, B., Veeck, L., Whiblye, T., Wilkes, C. and Wright, G. (1984) In vitro fertilization in Norfolk, Virginia, 19801983. Journal of in Vitro Fertilization and Embryo
Transfer, 1, 24-28. doi:10.1007/BF01129616

[15] Johnston, I., Lopata, A., Speirs, A., Hoult, I., Kellow, G. and Plessis, Y. (1981) In vitro fertilization: The challenge of the eighties. Fertility and Sterility, 36, 699-706.

[16] Kreiner, D., Muasher, S.J., Acosta, A.A., Jones, G.S., Liu, H.C. and Rosenwaks, Z. (1988) Monitoring gonadtropinstimulated cycles for in vitro fertilization and embryo transfer. Journal of in Vitro Fertilization and Embryo Transfer, 5, 230-233. doi:10.1007/BF01131127

[17] Laufer, N., DeCherney, A., Tarlatzis, Zuckerman, A.L., Polan, M.L., Dlugi, A.M., Graebe, R., Barnea, E.R. and Naftolin, F. (1984) Delaying hCG administration in human menopausal gonadotropin-induced cycles decreases successful IVF of human oocytes. Fertility and Sterility, 42, 198-203.

[18] Miller, K.F., Goldberg, J.M. and Falcone, T. (1996) Follicle size and implantation of embryos from in vitro fertilization. Obstetrics \& Gynecology, 88, 583-586. doi:10.1016/0029-7844(96)00241-4

[19] Nogueira, D., Friedler, S., Schachter, M., Raziel, A., RonEl, R. and Smitz, J. (2006) Oocyte maturity and preimplantation development in relation to follicular diameter in gonadotropin-releasing hormone agonist or antagonist treatments. Fertility and Sterility, 85, 578-583. doi:10.1016/i.fertnstert.2005.08.033

[20] O’Herlihy, C., Evans, J.H., Brown, J.B., de Crespigny, L. and Robinson, H. (1982) Use of ultrasound in monitoring ovulation induction with human pituitary gonadotropins. Obstetrics \& Gynecology, 60, 577-582.

[21] Rosen, M.P., Shen, S., Dobson, A.T., Rinaudo, P.F., McCulloch, C.E. and Cedars, M.I. (2008) A quantitative assessment of follicle size on oocyte developmental competence. Fertility and Sterility, 90, 684-690. doi:10.1016/j.fertnstert.2007.02.011

[22] Scott, R.T., Hofmann, G.E., Muasher, S.J., Acosta, A.A., Kreiner, D.K. and Rosenwaks, Z. (1989) Correlation of follicular diameter with oocyte recovery and maturity at the time of transvaginal follicular aspiration. Journal of in Vitro Fertilization and Embryo Transfer, 6, 73-75. doi:10.1007/BF01130729

[23] Tan, S.L., Balen, A., El Hussein, E.E., Mills, C., Campbell, S., Yovich, J. and Jacobs, H.S. (1992) A prospective randomized study of the optimum timing of hCG administration after pituitary desensitization in IVF. Fertility and Sterility, 57, 1259-1264.

[24] Wittmaack, F.M., Kreger, D.O., Blasco, L., Tureck, R.W., Mastroianni, L. and Lessey, B.A. (1994) Effect of follicular size on oocyte retrieval, fertilization, cleavage, and embryo quality in in vitro fertilization cycles: A 6-year data collection. Fertility and Sterility, 62, 1205-1210. 